\title{
New trends in intraocular lens imaging
}

\author{
María S. Millán ${ }^{* *}$, Francisco Alba-Bueno, Fidel Vega \\ Departament of Optics and Optometry, Technical University of Catalonia, \\ Terrassa (Barcelona), Spain
}

\begin{abstract}
As a result of modern technological advances, cataract surgery can be seen as not only a rehabilitative operation, but a customized procedure to compensate for important sources of image degradation in the visual system of a patient, such as defocus and some aberrations. With the development of new materials, instruments and surgical techniques in ophthalmology, great progress has been achieved in the imaging capability of a pseudophakic eye implanted with an intraocular lens (IOL). From the very beginning, optical design has played an essential role in this progress. New IOL designs need, on the one hand, theoretical eye models able to predict optical imaging performance and on the other hand, testing methods, verification through in vitro and in vivo measurements, and clinical validation. The implant of an IOL requires a precise biometry of the eye, a prior calculation from physiological data, and an accurate position inside the eye. Otherwise, the effects of IOL calculation errors or misplacements degrade the image very quickly. The incorporation of wavefront aberrometry into clinical ophthalmology practice has motivated new designs of IOLs to compensate for high order aberrations in some extent. Thus, for instance, IOLs with an aspheric design have the potential to improve optical performance and contrast sensitivity by reducing the positive spherical aberration of human cornea. Monofocal IOLs cause a complete loss of accommodation that requires further correction for either distance or near vision. Multifocal IOLs address this limitation using the principle of simultaneous vision. Some multifocal IOLs include a diffractive zone that covers the aperture in part or totally. Reduced image contrast and undesired visual phenomena, such as halos and glare, have been associated to the performance of multifocal IOLs. Based on a different principle, accommodating IOLs rely on the effort of the ciliary body to increase the effective power of the optical system of the eye in near vision. Finally, we present a theoretical approach that considers the modification of less conventional ocular parameters to compensate for possible refractive errors after the IOL implant.
\end{abstract}

Keywords: intraocular lens, cataract surgery, refractive error, human visual system, multifocal lens, diffractive lens, imaging efficiency

\section{INTRODUCTION}

Nowadays, cataract surgery can be seen as not only a rehabilitative operation, but a customized procedure to compensate for important sources of image degradation in the visual system of a patient, such as defocus and some aberrations. The surgical treatment involves the extraction and replacement of the crystalline by an intraocular lens (IOL). This work overviews the recent advances in IOL calculation, design, and testing. It also points out some ongoing tendencies that are being analyzed through optical testing and clinical studies.

\section{IOL POWER CALCULATIONS}

Assuming the thin lens equation in first-order approximation, the IOL power $\left(P_{I O L}\right)$ can be computed from the difference between the vergence of the exit or image beam $\left(X^{\prime}\right)$ and the vergence of the incident or object beam $(X)$, that is, $P_{I O L}=X^{\prime}-X$ [1]. This expression can be rewritten in terms of the refractive power of the cornea $K$, the distance between the corneal anterior vertex and the IOL, named the effective lens position ( $E L P$ ), and the axial length of the eye ( $A L X)$, which is the distance between the anterior vertex of the cornea and the retina (Fig. 1), to give

\footnotetext{
* millan@oo.upc.edu Tel 937398339 Fax 937398301
} 


$$
P_{I O L}=\frac{n_{v}}{A L X-E L P}-\frac{n_{q}}{\left(\frac{n_{q}}{K}\right)-E L P} .
$$

In Eq. $1, n_{q}$ and $n_{v}$ are the refractive indexes of aqueous and vitreous humors, respectively. In this equation, it is assumed that $K$ and $A L X$ can be measured, and $E L P$ can be predicted with enough precision. Even in the simple paraxial approach, this basic optical formula has encountered many practical difficulties in application for a number of reasons concerning in vivo biometry, eye model-based formulas and interconnected nuances. These difficulties include the need to make assumptions on, for example, the thin or thick lens model to calculate the cornea and lens powers, the refractive indexes, the correction of principal planes of a thick lens model, the prediction of the final postoperative position of the IOL, the correction of the axial length for retinal thickness, and the various axes of the eye. A different statistical approach has led to formulas empirically derived from the regression analysis of a large number of cases. These formulas (first represented by SRK formulas, being SRK/T [2] the latest version) incorporate some constants according to the type of IOL and the mean values of different biometric parameters, such as $K$ and $A L X$ readings and the corneal diameter. The formula for IOL power calculation [3], [4] can be made additionally sensitive to differences in a number of practice-specific variables, such as the surgical technique, unusual axial lengths typified as short and long eyes, etc. The interested reader is referred to Refs. [5-8] further insight in the field. Although the majority of methods used in clinical practice over the past three decades have been based on a regression approach, the recent improvements in diagnostic equipment and the advances in surgical technology are giving the relevance back to the formulas based on optical imaging, which have been proved to be more accurate and robust to changes in the clinical environment.

Patients who have undergone corneal refractive surgery present an additional difficulty for the calculation of IOL power. The changes introduced in the cornea makes the calculation of the corneal power more difficult and, hence, the calculation of IOL power. Several methods have been proposed to tackle these cases taking into consideration that preoperative data may or may not be available. They have been reviewed by Savini et al. [9].

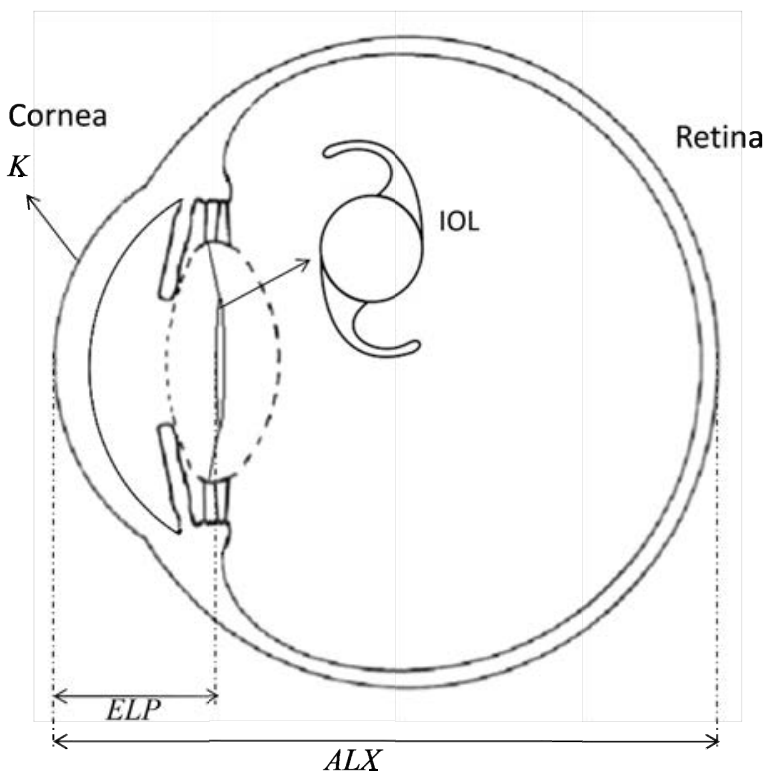

Figure 1. Schematic of an eye implanted with an intraocular lens. The dashed line shows the former position of the crystalline. See the text (Sec. 2) for the meaning of abbreviations. 


\section{HIGH ORDER ABERRATION COMPENSATION}

The first models of the eye only considered spheres to describe all the optical surfaces operating in paraxial approach (first-order Gaussian optics). Despite its many limitations and simplifications, the Gullstrand four-spherical surface model [10] has been long used to generically describe the imaging optical design of the eye and, in this context, it has been also taken as an early reference to describe a pseudophakic eye implanted with an IOL. However, it is known that for the cornea, a conic surface model is more realistic. Ray tracing computations from corneal topographic data have permitted the characterization of the natural high order aberrations existing in the cornea [11]. On average, the cornea introduces significant amounts of aberrations into the optical system of the eye, mainly astigmatism, coma, and spherical aberration. These aberrations are compensated in part by internal optics, particularly the crystalline lens, so that total values measured for the optical system of the eye are lower than for the cornea alone [12-15]. Regarding spherical aberration (SA), the average human cornea shows a prolate like shape that, in terms of the $c[4,0]$ Zernike coefficient, produces a natural positive SA of about $0.3 \mu \mathrm{m}$ for a pupil diameter of $6 \mathrm{~mm}$ [16]. These facts led to a new design for the IOL that, analogously to the young crystalline lens, introduced opposite aberrations to that of the cornea. The initial proposal for the use of an aspheric IOL design [17] was renewed a decade later in the context of the compensation of corneal aberration and thus, the optical element (aspheric IOL) was manufactured and reached the surgical practice [18], [19]. Numerous studies have compared the aberrations in eyes implanted with aspheric and spherical IOLs using theoretical analysis, experimental results obtained in eye models and postoperative results. While a better in-focus performance corresponds to the aspheric IOL, a significantly lower optical depth of field is obtained with this lens as well [20]. This effect becomes a disadvantage for those eyes whose accommodation capability turns out to be seriously affected after the implant (we will come back to this issue in Section 5).

Not only does the implant of an IOL require a precise calculation but also an accurate position inside the eye. Otherwise, the effects of IOL misplacements degrade the image very quickly. The effects of decentration and tilt of the IOL have been discussed in [17] and [19] based on theoretical models. Their impact on the imaging quality is more deleterious in aspheric than spherical [17]. Decentration of aspheric lenses is more critical than tilt [21]. In contrast to an aspheric IOL with negative SA designed to offset the positive SA of the average cornea, the so-called aberration-free IOL has no SA [22]. The latter has proved to be more resistant to decentration and tilt than the former, and still provides better image quality than spherical IOLs [23],[24]. Between the aberration-compensating and the aberration-free IOLs, a variety of aspheric lenses with intermediate SA values are currently available for clinical practice.

In this line of research, a more recent design of IOL aims to correct the coma generated by the angular misalignments existing between the different optical components of the eye or, in other words, by the lack of a common optical axis. Coma-compensating IOL has been proposed in [25] taking into consideration off-axis conditions, a cornea with an average asphericity [25], [19], and the theoretical model eye described by Holladay et al in [19].

\section{MODEL EYES FOR IOL TESTING}

New IOL designs need, on the one hand, theoretical eye models able to predict optical imaging performance and on the other, testing methods, verification through in vitro and in vivo measurements [26], and clinical validation [27]. The literature shows a great variety of models of each component (cornea, lens) and from them models that describe the whole optical system of the eye. Many of them have been extensively reviewed in [10], [28] and [29].

As stated in [30] an imaging quality criterion for intraocular lenses should be related to the optics of the eye. The International Organization for Standardization (ISO) proposed a model eye with a cornea free of aberration so that any measured aberration would be due to the IOL [31]. But the ISO eye model was conceived before the introduction of aspheric IOLs. As described in Section 3, there are IOLs that are purposely designed with negative SA to compensate for the natural positive SA measured in normal human corneas. When these IOLs are tested in the ISO-based model eye, a paradoxical worse performance than for conventional spherical IOLs with positive SA is obtained. The current ISO standard is under revision [32]; for the next edition, an eye model with an artificial cornea that would reproduce the level of the SA of the average human cornea is being considered. Some modifications of the current ISO eye model as well as a practical realization called "physiological model eye," with dimensions close to the natural eye have been proposed and analysed in [33]. An aspheric lens used as an artificial cornea is expensive and difficult to manufacture. Instead, a simple biconvex spherical lens of the same focal length and similar wavefront error can be advantageously used for the on-axis optical assessment of IOLs as it has been demonstrated in [34]. 
An experimental realization of an eye model on optical bench for IOL imaging quality assessment is sketched in Figure 2 [35]. The LED source with the test object and the collimating lens produce the object beam that illuminates the model eye (formed by the iris diaphragm, the artificial cornea and the wet cell) where the IOL is inserted. The IOL's holder is mounted in high precision linear ( $\mathrm{X}, \mathrm{Y}$ and $\mathrm{Z}$ axis) and rotation (tilt and tip) stages. The test object is imaged by the model eye with the IOL in the plane that resembles the retina. An infinite corrected microscope mounted in a translation holder is used to image the retinal plane onto a CCD camera with magnification. This setup has been designed to meet the conditions fixed by the ISO standard with the additional possibility of using artificial corneas with different amount of SA for the assessment of aspheric IOLs. The setup of Figure 2 permits to analyze the optical performance of an IOL in a variety of circumstances: on- and off- axis, under controlled decentration and/or tilt, under different illuminating wavelengths or white light, for a object point (pinhole test) or for a wide field object (e.g. USAF test, grids). A software application allows the computation of conventional metrics (such as point spread function, modulation transfer function, and Strehl ratio). The camera can be replaced by either a spectrofotometer for spectral analysis or a Hartmann-Shack sensor for wavefront analysis through expansion in Zernike polynomials.

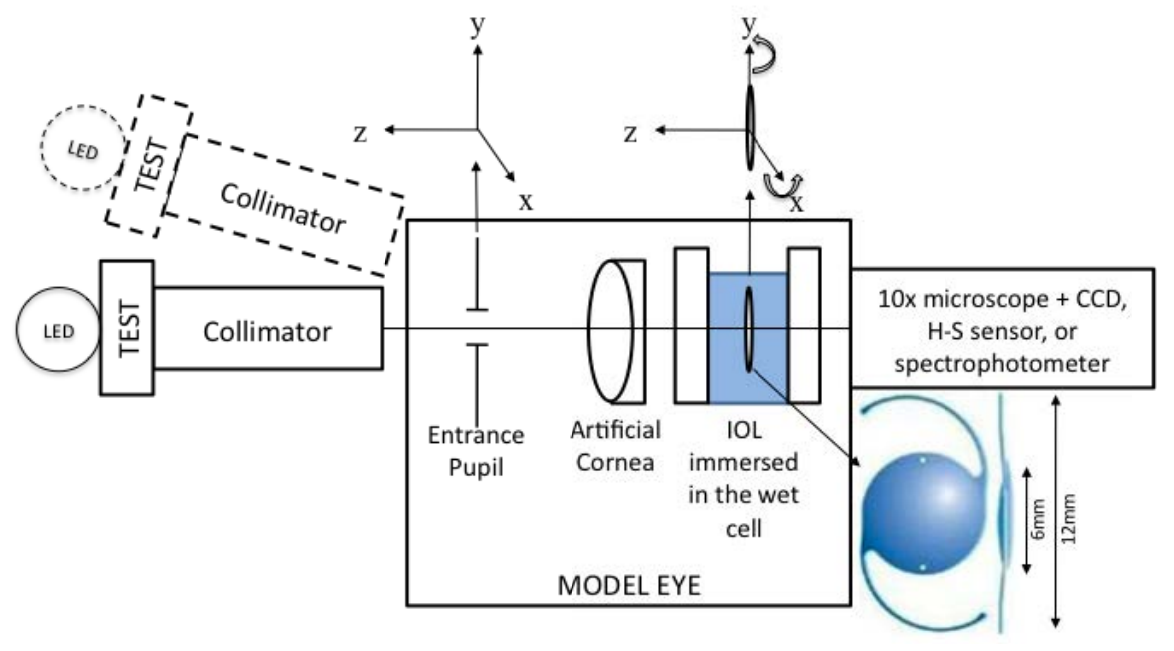

Figure 2. Sketch of an experimental realization of an eye model for IOL imaging quality assessment on optical bench.

A different approach consists of building a computer model of an IOL implanted eye from accurate biometric data. From individual measurements of post-operative corneal topography, anterior chamber depth, IOL geometry, IOL tilts and decentration, the misalignment of the line of sight, and using an optical design program as specific software application, a customized model of pseudophakic eye can be built [36]. This method has been validated by comparing the estimates of aberrations obtained from numerical ray tracing using the customized model of eye with the experimental measurements of ocular aberrations in real pseudophakic eyes.

\section{MULTIFOCAL IOL FOR DISTANCE AND NEAR IMAGING}

The crystalline lens performance is controlled by the surrounding ciliary muscles that, in a young eye, allow the crystalline lens to bend, flex, thicken, and thin to focus images on the retina as the observer look at objects near and far. This process is called accommodation. As the eye ages, its lens loses flexibility and becomes less accommodating. It can also become clouded with cataracts and require from surgery to replace the crystalline by an implant. So far, monofocal IOLs can only provide clear vision for a very limited depth of field and require surgeons to correct either distance or near vision by means of additional corrective lenses. Multifocal IOLs are designed to reduce the dependence on spectacles. They address the lack of accommodation using the principle of simultaneous vision. The multifocal lens ussually has two powers that focus the incoming light on two separated planes, one for distance vision and the other one for near vision. Since near and distance images are superimposed on the retina, brain adaptation plays an important role. It must choose between the two images based on the object at which the observer is looking. For a distant object, a sharp retinal image is 
provided by the parts of the IOL within the pupillary area that have the distance correction and, simultaneously at the same place, a somewhat blurred and scaled image is provided by the other parts of the IOL. The decrease in contrast of the in-focus image is caused by the energy distribution between the distance and near focus. The superposition of the infocus and out-of-focus images, of different scale, on the retina may lead to a rivalry or confusion, often associated with other unpleasant visual fenomena, such as glare and halos, in mesopic and escotopic conditions. This situation, however, can be overcome by the brain's capability to adapt itself to multifocality.

Two basic designs have been proposed to improve the performance of these lenses by directing different amounts of light to the distance or near images depending on pupil diameter [37]: the so-called refractive and diffractive multifocal IOLs. A refractive multifocal IOLs is a multizone multifocal lens, where the different concentric refractive areas have also different width. Recently, a refractive multifocal IOL with rotational asymmetry has been developed and introduced into clinical practice. Their performance is being evaluated and compared with already existing IOL designs [37-39]. A diffractive multifocal IOL uses the base lens curvature and the zero and first diffraction orders to simultaneously produce the two focal points [40], [41]. While the power corresponding to the zero diffraction order is used to image distance objects, the other is used for near vision. An apodized diffractive profile, limited to the central area of the IOL, has been proposed to obtain an increasing distance-dominant performance for large pupils. According to its theoretical design, the apodized diffractive multifocal IOL varies the energy balance between the distance and near image as a function of the eye pupil [42]. In addition to this, some of these lenses have an aspheric base design that can be advantageous in comparison the conventional spherical one. There is a great interest to determine either in a test bench [43], [44] or in clinical studies [45], [46] the conditions and the degree to which the aspheric design in apodized diffractive multifocal IOLs might be advantageous versus a conventional spherical design, particularly when some studies have shown little or no benefit of aspheric IOLs with small pupils [47, [48]. For the assessment of the optical performance of multifocal IOLs, the common metrics (point spread function, modulation transfer function, and visual acuity) have to be measured [38], [49-52] and also its behaviour in terms of the energy balance between the distance an near images and its variation with the pupil diameter. Very recent studies carried out in an optical bench show a discrepancy between the theoretical prediction of the design and the experimentally measured energy distribution between the two foci in apodized diffractive multifocal IOLs [53]. For large pupils, the energy efficiency of the distance image is strongly affected by the level of SA, which was no predicted by the original design. For small pupils, there are no significant differences between the aspheric and the spherical multifocal IOLs .

A different approach for distance and near imaging aims to achieve an IOL's true accommodation function, which involves transient and reversible changes of two kinds: axial displacement of the IOL and/or increased optical power of the IOL [54]. Accommodating IOLs rely on the capability of ciliary body to keep its activity even after the extraction of the crystalline lens. The simplest design for accommodating IOL is a fixed-power single lens with a flexible haptic support system that permits axial displacements of the lens during the accommodative effort. Another design combines two lenses in one (dual-optic accommodating IOL [55]. It consists of a plus-powered lens in the front and a minus powered lens in the back that are joined by spring haptics. When the two lenses are close together, the eye should be set for distance vision. As the ciliary body contracts, this takes tension off of the zonules, the capsular bag relaxes and the lenses are allowed to spring apart. In this condition the eye should be set for near vision.

\section{MOVING FORWARD}

According to Olsen in [5], despite the efforts to target IOL power calculations for a refractive prediction with an absolute error lower than $0.5 \mathrm{D}$, about $90 \%$ of cases fall within $\pm 1.0 \mathrm{D}$ and $99.9 \%$ within $\pm 2.0 \mathrm{D}$ of their targets. Higher errors are obtained in long and short eyes and patients that have undergone prior CRS. The blur due to defocus establishes the narrowest limits for acceptability. The defocus associated with a refractive error ranging in absolute value from $0.5 \mathrm{D}$ to 1.0D has been classified from troublesome to objectionable (for a $5 \mathrm{~mm}$ effective pupil and black letter targets) [56]. Consequently, some post-surgical refractive errors may require further neutralization. To this end, corneal ablation to modify the curvature of the anterior surface of the cornea can be effective. This is in fact the preferred option in many cases, but it is not always recommendable or even possible. For instance, many patients that currently need cataract surgery underwent corneal ablative surgery in their eyes some time ago. It is not unusual to find cases that are the result of a not very refined application of the ablation technique at its early stages, or cases for which the resulting corneal thickness would not admit a second ablation in safe conditions. A possible solution can be found in the use of light- 
adjustable IOL that is adjusted using safe levels of ultraviolet light [57]. After implantation, the lens power may be increased or decreased noninvasively by the application of the appropriate spatially resolved irradiation profile.

The modification of other ocular parameters to compensate for possible refractive error after the IOL implant has been analysed as a hypothesis in [8]. The analysis aims to determine which components of the eye would be the most advantageous to modify provided the necessary biocompatible materials were available. The preliminary results show that changes in the corneal refractive index, thickness or posterior radius of curvature have relatively little effect on the overall refractive error. However, small changes in the refractive indexes of the aqueous or the vitreous humors are highly effective, much more than a similar amount of change in the anterior curvature of the cornea. This fact opens new and attractive possibilities to compensate for defocus, through the introduction of changes in degrees of freedom that have been considered unconventional up until now. These results agree with the results formerly obtained by Atchison and Smith for the Gullstrand eye [10]. Although some questions arise concerning biocompatibility, stability, and drainage, the great progress experienced in the generation of new materials allows us to consider this proposal at the basis of promising upcoming techniques.

\section{CONCLUSIONS}

The recent developments of IOLs overviewed in this work show a very active multidisciplinary field of research with important implications in visual health care. The optical design is essential to optimize the optical imaging quality of an IOL, but at the same time, it requires the parallel improvement of some collaborative techniques: biological data acquisition through more precise instruments, eye modeling to obtain more accurate, complete and customized models of eye, new biocompatible materials with proper opto-mechanical features, more refined surgical techniques, and more complete methods for the evaluation post-operative results. The recent and future achievements obtained in this field of research will benefit not only patients suffering from cataracts, but a more general population with age over fifty whose eye lenses progressively lose transparency and the capability to accommodate.

\section{ACKNOLEDGEMENTS}

This research has been supported by Spanish Ministerio de Educación y Ciencia and FEDER funds under project No. DPI2009-08879.

\section{REFERENCES}

[1] Fyodorov, S.N. and Kolonko, A.I. "Estimation of optical power of the intraocular lens," Vestnik Oftalmologic (Russian) (Moscow) 4, 27-31 (1967).

[2] Sanders, D.R., Retzlaff, J.A., Kraff M.C., Gimbel, H.V. and Raanan M.G., "Comparison of the SRK/T formula and other theoretical and regression formulas," J Cataract Refract Surg 16, 341-346 (1990).

[3] Holladay J.T., "Consultations in refractive surgery," J Cataract Refract Surg 5, 202-203 (1989).

[4] Hoffer K.J., "The Hoffer Q formula: a comparison of theoretic and regression formulas," J Cataract Refract Surg 19, 700-712 (1993).

[5] Olsen, T., "Calculation of intraocular lens power: a review," Acta Ophthalmol Scand 85(5), 472-485 (2007).

[6] Holladay, J.T., "Intraocular lens power calculations: correction of defocus," Chapter 4 in Refractive Lens Surgery, I. Howard Fine, Mark Packer, Richard S. Hoffmann, Eds. Springer, Berlin Heidelberg New York, 21-38 (2005).

[7] Fang, J.P., Hill, W., Wang, L., Chang, V. and Koch, D. D. “Advanced intraocular lens power calculations,” Chapter 4 in Cataract and Refractive Surgery, Th. Kohnen, D. D. Koch, Eds, Springer, Berlin Heidelberg, 31-46 (2006).

[8] Alba-Bueno, F. and Millán, M. S., "Defocus correction in the optical system of the eye: unconventional degrees of freedom," Journal of Biomedical Optics 16(1), 016010 (2011).

[9] Savini, G., Barboni, P. and Zanini, M., "Intraocular lens power calculation after myopic refractive surgery: theoretical comparison of different methods," Ophthalmology 113: 1271-1282 (2006).

[10] Atchison, D. and Smith, G., Optics of the Human Eye, Butterworth-Heinemann, Oxford (2000). 
[11] Schwiegerling, J., Greivenkamp, J.E., "Using corneal height maps and polynomial decomposition to determine corneal aberrations". Optom Vis Sci. 74, 906-916 (1997).

[12] Millodot, M,, Sivak, J., "Contribution of the cornea and lens to the spherical aberration of the eye," Vision Res 19, 685-687 (1979).

[13] Navarro, R., Santamaría, J. and Bescós, J., “Accommodation-dependent model of the human eye with aspherics," J Opt Soc Am A. 1985;2:1273-1281.

[14] Artal, P., Guirao, A., Berrio, E. and Williams, D. R., "Compensation of corneal aberrations by the internal optics in the human eye," J Vis 1, 1-8 (2001).

[15] Kelly, J. E., Mihashi, T., Howland, H. C., "Compensation of corneal horizontal/vertical astigmatism, lateral coma, and spherical aberration by internal optics of the eye," J. Vision 4 (4), 262-271 (2004).

[16] Norrby, S., Piers, P., Campbell Ch. and van der Mooren, M., "Model eyes for evaluation of intraocular lenses," Applied Optics 46(26), 6595-6605 (2007).

[17] Atchison, D. A., "Design of aspheric intraocular lenses," Ophthalmic Physiol. Opt. 11, 137-146 (1991).

[18] Guirao, A., Redondo, M., Geraghty, E., Piers, P., Norrby, S. and Artal, P. "Corneal optical aberrations and retinal image quality in patients in whom monofocal intraocular lenses were implanted," Arch. Ophthalmol. 120, 11431151 (2002).

[19] Holladay, J. T., Piers, P. A., Koranyi, G., van der Mooren, M. and Norrby, N. E. "A new intraocular lens design to reduce spherical aberration of pseudophakic eyes,” J. Refract. Surg 18 (6), 683-691 (2002).

[20] Marcos, S. Barbero, S. and Jiménez-Alfaro, I., "Optical quality and depth-of-field of eyes implanted with spherical and aspheric intraocular lenses," J. Refract. Surg 21 (3), 223-235 (2005)

[21] Tabernero, J., Piers, P., Benito, A., Redondo, M., Artal, P., "Predicting the optical performance of eyes implanted with IOLs to correct spherical aberration," Invest Ophthalmol Vis Sci 47(10), 4651-4658 (2006).

[22] Altmann, G.E., “Aspheric lenses and lens family,” U.S. Patent US2005/0203619 A1 (2005)

[23] Altmann, G.E., Nichamin, L. D., Lane, S.S and Pepose, J.S. "Optical performance of 3 intraocular lens designs in the presence of decentration," J Cataract Refract Surg 31(3), 574-585 (2005).

[24] Eppig, T., Scholz, K., Loffler, A., Meßner, A. and Langenbucher, A., "Effect of decentration and tilt on the image quality of aspheric intraocular lens designs in a model eye," J Cataract Refract Surg 35(6); 1091-1100 (2009).

[25] Tabernero, J., Piers, P. and Artal, P., “Intraocular lens to correct corneal coma," Opt. Lett. 32(4), 406-408 (2007).

[26] Barbero, S., Marcos, S. and Jiménez-Alfaro, I., "Optical aberrations of intraocular lenses measured in vivo and in vitro," J. Opt. Soc. Am 20 (10), 1841-1851 (2003).

[27] Norrby, S. "Sources of error in intraocular lens power calculation," J. Cataract Refract. Surg 34 (3), 368-376 (2008).

[28] Navarro, R., "The optical design of the human eye: a critical review," J. Optom. 2(1), 3-18 (2009).

[29] Artal, P., Tabernero, J., "Optics of human eye: 400 years of exploration from Galileo's time," Applied Optics 49 (16), 123-130 (2010).

[30] Norrby, S., "Standarized methods for assessing the imaging quality of intraocular lenses," Applied Optics 34 (31), 7327-7333 (1995).

[31] International Organization for Standardization (ISO), ISO 11979-2 Ophthalmic Implants, Intraocular Lenses Part 2: Optical Properties and Test Methods (ISO, 1999).

[32] Norrby, S., "ISO eye model not valid for assessing aspherical lenses," J. Cataract. Refract. Surg. 33(12), 10561057 (2008)

[33] Norrby, S., Piers, P., Campbell, Ch. and van der Mooren, M., "Model eyes for evaluation of intraocular lenses," Applied Optics 46(26), 6595-6605 (2007).

[34] F. Vega, M. S. Millán, and B. Wells, "Spherical lens versus aspheric artificial cornea for intraocular lens testing," Opt. Lett. 35(10), 1539-1541 (2010) .

[35] Alba-Bueno, F., Vega, F. and Millán, M. S. "Design of a test bench for intraocular lens optical characterization," Journal of Physics: Conference Series 274, 012105-012111 (2011).

[36] Rosales, P. and Marcos, S., "Customized computer models of eyes with intraocular lenses," Optics Express 15(5), 2204-2218 (2007)

[37] Lane, S. S., Morris, M., Nordan, L., Packer, M., Tarantino, N. and Wallace, R. B., "Multifocal Intraocular lenses," Ophthalmol Clin N Am 19(1), 89-105 (2006). 
[38] Alió, J. L.,Piñero, D P., Plaza-Puche, A.B. and Rodriguez Chan, M. J., "Visual outcomes and optical performance of a monofocal intraocular lens and a new-generation multifocal intraocular lens," J Cataract Refract Surg 37(2),241-250 (2011).

[39] McAlinden, C. and Moore, J. E., "Multifocal intraocular lens with a surface-embedded near section: Short-term clinical outcomes," J Cataract Refract Surg 37 (3), 441-445 (2011).

[40] Larsson, M., Beckman, C., Nyström, A., Hård, S. and Sjöstrand, J., "Optical properties of diffractive, bifocal, intraocular lenses," Appl. Opt. 31, 2377-2384 (1992).

[41] Cohen, A. L., "Practical design of a bifocal hologram contact lens or intraocular lens," Appl. Opt. 31, 3750-3754 (1992).

[42] Davison, J.A. and Simpson, M. J., "History and development of the apodized diffractive intraocular lens," J Cataract Refract Surg 32, 849-858 (2006).

[43] Pieh, S., Marvan, P., Lackner, B., Hanselmayer, G.,Schmidinger, G., Leitgeb, R., Sticker, M., Hitzenberger, C. K., Fercher, A. F., et al., "Quantitative Performance of Bifocal and Multifocal Intraocular Lenses in a Model Eye: Point Spread Function in Multifocal Intraocular Lenses," Arch Ophthalmol 120, 23-28 (2002).

[44] Terwee, T., Weeber, H., van der Mooren, M. and Piers, P., "Visualization of the retinal image in an eye model with spherical and aspheric, diffractive, and refractive multifocal intraocular lenses," J Refract Surg 24, 223-232 (2008).

[45] Kohnen, T., Klaproth, O. K. and Bu hren, J., "Effect of intraocular lens asphericity on quality of vision after cataract removal: An intraindividual comparison," Ophthalmology 116, 1697-1706 (2009).

[46] de Vries,N. E., Webers,C. A., Verbakel, F., de Brabander, J.,. Berendschot, T. T, Cheng, Y. Y., Doors, M. and Nuijts, R.M. "Visual outcome and patient satisfaction after multifocal intraocular lens implantation: aspheric versus spherical design," J Cataract Refract Surg 36, 1897-1904 (2010).

[47] Muñoz, G., Albarran-Diego, C., Montes-Micó, R., Rodriguez-Galietero, A. and Alio, J. L., "Spherical aberration and contrast sensitivity after cataract surgery with the Tecnis Z9000 intraocular lens," J Cataract Refract Surg 32, 1320-1327 (2006).

[48] Kasper, T., Bu hren, J. and Kohnen, T. "Visual performance of aspheric and spherical intraocular lenses: Intraindividual comparison of visual acuity, contrast sensitivity, and higher order aberrations," J. Cataract Refract Surg 32, 2022-2029 (2006).

[49] Eppig, T., Scholz, K. and Langenbucher, A., "Assessing the optical performance of multifocal (diffractive) intraocular lenses," Ophthalmic Physiol Opt 28, 467-474 (2008).

[50] Pieh, S., Fiala, W., Malz, A. and Stork, W., "In Vitro Strehl Ratios with Spherical, Aberration-Free, Average, and Customized Spherical Aberration-Correcting Intraocular Lenses," Investigative Ophthalmology \& Visual Science 50, $1264-1270$ (2009).

[51] Castignoles, F., Flury, M. and Lepine, T., "Comparison of the efficiency, MTF and chromatic properties of four diffractive bifocal intraocular lens designs," Opt Express 18,5245-5256 (2010).

[52] Weeber, H. A., Featherstone, K. A. and Piers, P. A., "Population-based visual acuity in the presence of defocus well predicted by classical theory," J. Biomedical Optics Letters 15(4), 040509-3p. (2010).

[52] Vega, F., Alba-Bueno, F. and Millán, M. S., "Energy distribution between distance and near images in apodized diffractive multifocal intraocular lenses," Investigative Ophthalmology \& Visual Science (accepted 2011, in press).

[54] Masket, S., "Accommodating IOLs: Emerging concepts and designs," Cataract and Refractive Surgery Today, July 2004.

[55] McLeod, S. D., "Optical principles, biomechanics, and initial clinical performance of a dual-optic accommodating intraocular lens," Trans Am Ophthalmol Soc 104,437-452 (2006).

[56] Atchison, D.A., Guo, H., Charman, W.N. and Fisher S.W., "Blur limits for defocus, astigmatism and trefoil," Vision Research 49, 2393-2403 (2009).

[57] Schwartz, D.M., "Light-adjustable lens: development of in vitro nomograms," Trans Am Ophthalmol Soc 102, 67-74 (2004). 